\title{
Fick versus flow: a real-time invasive cardiovascular magnetic resonance (iCMR) reproducibility study
}

\author{
Yousef Arar ${ }^{1,3^{*}}$ (D), Tarique Hussain ${ }^{1,2,3}$, Riad Abou Zahrr ${ }^{1,3}$, Vasu Gooty ${ }^{1,3}$, Joshua S. Greer ${ }^{1}$, Rong Huang ${ }^{4}$,
} Jennifer Hernandez ${ }^{5}$, Jamie King ${ }^{1,3}$, Gerald Greil ${ }^{1,2,3}$ and Surendranath R. Veeram Reddy ${ }^{1,3}$

\begin{abstract}
Background: Cardiac catheterization and cardiovascular magnetic resonance (CMR) imaging have distinct diagnostic roles in the congenital heart disease (CHD) population. Invasive CMR (iCMR) allows for a more thorough assessment of cardiac hemodynamics at the same time under the same conditions. It is assumed but not proven that iCMR gives an incremental value by providing more accurate flow quantification.
\end{abstract}

Methods: Subjects with CHD underwent real-time 1.5 T iCMR using a passive catheter tracking technique with partial saturation pulse of $40^{\circ}$ to visualize the gadolinium-filled balloon, CMR-conditional guidewire, and cardiac structures simultaneously to aid in completion of right (RHC) and left heart catheterization (LHC). Repeat iCMR and catheterization measurements were performed to compare reliability by the Pearson (PCC) and concordance correlation coefficients (CCC).

Results: Thirty CHD (20 single ventricle and 10 bi-ventricular) subjects with a median age and weight of 8.3 years (2-33) and $27.7 \mathrm{~kg}$ (9.2-80), respectively, successfully underwent iCMR RHC and LHC. No catheter related complications were encountered. Time taken for first pass RHC and LHC/aortic pull back was 5.1, and 2.9 min, respectively. Total success rate to obtain required data points to complete Fick principle calculations for all patients was 321/328 (98\%). One patient with multiple shunts was an outlier and excluded from further analysis. The PCC for catheter-derived pulmonary blood flow (Qp) $(0.89, p<0.001)$ is slightly lower than iCMR-derived Qp $(0.96, p<0.001)$, whereas catheterderived systemic blood flow (Qs) $(0.62, p=<0.001)$ was considerably lower than iCMR-derived Qs $(0.94, p<0.001)$. CCC agreement for Qp at baseline (C1-CCC $=0.65,95 \% \mathrm{Cl} 0.41-0.81$ ) and retested conditions (C2-CCC $=0.78$, $95 \% \mathrm{Cl} 0.58-0.89)$ were better than for $\mathrm{Qs}$ at baseline $(\mathrm{C} 1-\mathrm{CCC}=0.22,95 \% \mathrm{Cl}-0.15-0.53)$ and retested conditions (C2-CCC $=0.52,95 \% \mathrm{Cl} 0.17-0.76)$.

Conclusion: This study further validates hemodynamic measurements obtained via iCMR. iCMR-derived flows have considerably higher test-retest reliability for Qs. iCMR evaluations allow for more reproducible hemodynamic assessments in the CHD population.

Keywords: Congenital heart disease, Interventional CMR, Cardiac catheterization, Magnetic resonance imaging, Device tracking, Reproducibility

*Correspondence: Yousef.Arar@UTSouthwestern.edu

3 Pediatric Cardiology, Children's Medical Center, 1935 Medical District Drive, Dallas, TX 75235, USA

Full list of author information is available at the end of the article

\section{Background}

Non-invasive cardiovascular magnetic resonance (CMR) and invasive cardiac catheterization have distinct diagnostic roles in the congenital heart disease (CHD) original author(s) and the source, provide a link to the Creative Commons licence, and indicate if changes were made. The images or other third party material in this article are included in the article's Creative Commons licence, unless indicated otherwise in a credit line to the material. If material is not included in the article's Creative Commons licence and your intended use is not permitted by statutory regulation or exceeds the permitted use, you will need to obtain permission directly from the copyright holder. To view a copy of this licence, visit http://creativecommons.org/licenses/by/4.0/. The Creative Commons Public Domain Dedication waiver (http://creativeco mmons.org/publicdomain/zero/1.0/) applies to the data made available in this article, unless otherwise stated in a credit line to the data. 
population [1, 2]. CMR contributes important volume and flow data while cardiac catheterization allows for direct pressure and saturation measurements. By linking these powerful modalities, invasive CMR (iCMR) allows for a more accurate and thorough assessment of cardiac hemodynamics at the same time under the same conditions. Accurate pulmonary vascular resistance (PVR) and systemic blood flow measurements are critical for clinical decision making.

In this study, we describe a hemodynamics reproducibility comparison of catheter-based Fick principle measurements (equations outlined below) and CMRderived pulmonary (Qp) and systemic (Qs) blood flows. Fick principle calculations require measurement of oxygen consumption $\left(\mathrm{VO}_{2}\right)$ and hemoglobin $(\mathrm{Hg})$, as well as obtaining blood saturations in specific locations throughout the body (pulmonary veins $=\mathrm{PV}$, pulmonary artery $=\mathrm{PA}$, mixed venous $=\mathrm{MV}$ ). PVR and systemic vascular resistance (SVR) can then be determined by obtaining pressure measurements across the pulmonary bed (transpulmonary pressure gradient (TPG)) and systemic circulation (transsystemic pressure gradient (TSG)). via the bedside PRiMEGen system [National Institutes of Health, Bethesda, Maryland, USA] [4] during the iCMR procedure. This system feeds into a standard hemodynamic recording system (Sensis, Siemens Healthineers, Erlangen, Germany). This recording, together with the real-time CMR images, is projected in-room by using a shielded projector system [standard projector system for in-room Ambient system ambiance (Philips Healthcare, Best, the Netherlands)] (Fig. 1E).

\section{MR-conditional catheter and guidewire (Fig. 1C)}

A 6-French Arrow, Balloon Wedge-Pressure Catheter (Model AI-07124 and/or AI-07126, Teleflex, Wayne, Pennsylvania, USA) was used for all procedures. The balloon-tip of the catheter was filled with dilute gadolinium (1-part gadolinium to 99-parts saline) and guided with the help of an MR-conditional guidewire to specific structures in the heart to obtain necessary hemodynamics. This 0.035 " guidewire is United States Food and Drug Administration (FDA) cleared and Conformité Européenne (CE) marked MR-conditional guidewire (angled-tip Emeryglide MRWire, Nano4Imaging, Aachen, Germany).

$$
Q p=\frac{V O 2}{13.6 x H g x(P V-P A S a t)} Q s=\frac{V O 2}{13.6 x H g x(\text { systemic }-M V S a t)} \quad P V R=\frac{T P G}{Q p} S V R=\frac{T S G}{Q s}
$$

All catheter-based measurements were performed under real-time CMR imaging to guide a gadoliniumfilled balloon and an MR-conditional guidewire without the use of ionizing radiation.

\section{Methods}

\section{Study population}

The protocol was approved by the Institutional Review Board (IRB) (STU 032017-061) and was performed in the Children's Health CMR catheterization suite at Children's Medical Center (CMC) in Dallas, Texas, USA. In this protocol, we conducted heart catheterization using realtime CMR in pediatric and adult subjects already undergoing clinically indicated heart catheterization for CHD. In many subjects, CMR was also clinically indicated. If CMR was not required for clinical purposes, only CMR function and flow measurements were acquired as a research procedure. After discussing the indications for and risks of the procedure, prospective informed consent and assent were obtained from all subjects and/or legal guardians as appropriate for all study related procedures.

\section{iCMR environment and equipment (Fig. 1)}

Our iCMR environment and equipment has been previously described in detail [3]. Briefly, the interventionalist has direct visualization of catheter-derived pressures
Left heart catheterization (LHC) was performed with a 4-French balloon wedge or a 4-French non-braided pigtail catheter (Soft-Vu, Angiodynamics, Latham, New York, USA). The femoral artery sheath was upsized to 6-French only when the MR-conditional wire was necessary.

\section{Interventionalist timeout and access (zone 3)}

Once anesthesia induction is complete, the groin and/or neck are sterilely prepared and draped. The iCMR team performs a separate timeout. A baseline $\mathrm{VO}_{2}$ is measured and recorded for catheter-based Fick method calculations. If the subject was $<15 \mathrm{~kg}, \mathrm{VO}_{2}$ was assumed based on published references [5]. Three patients included in this study were $<15 \mathrm{~kg}$ at the time of their procedure. Percutaneous entry with short sheaths are placed in the femoral vein (6-French), femoral artery (4-French), and, if necessary, internal jugular vein (6-French) with ultrasound guidance. The 4-French sheath was upsized to a 6-French sheath whenever necessary to accommodate the $0.035^{\prime \prime}$ MR-conditional guidewire. At this time, an initial blood gas and activated clotting time were drawn to establish subject baseline and appropriateness to proceed. Heparin is administered for anticoagulation once access is complete. All iCMR procedures were performed with the patient at steady-state (in room air-21\% 


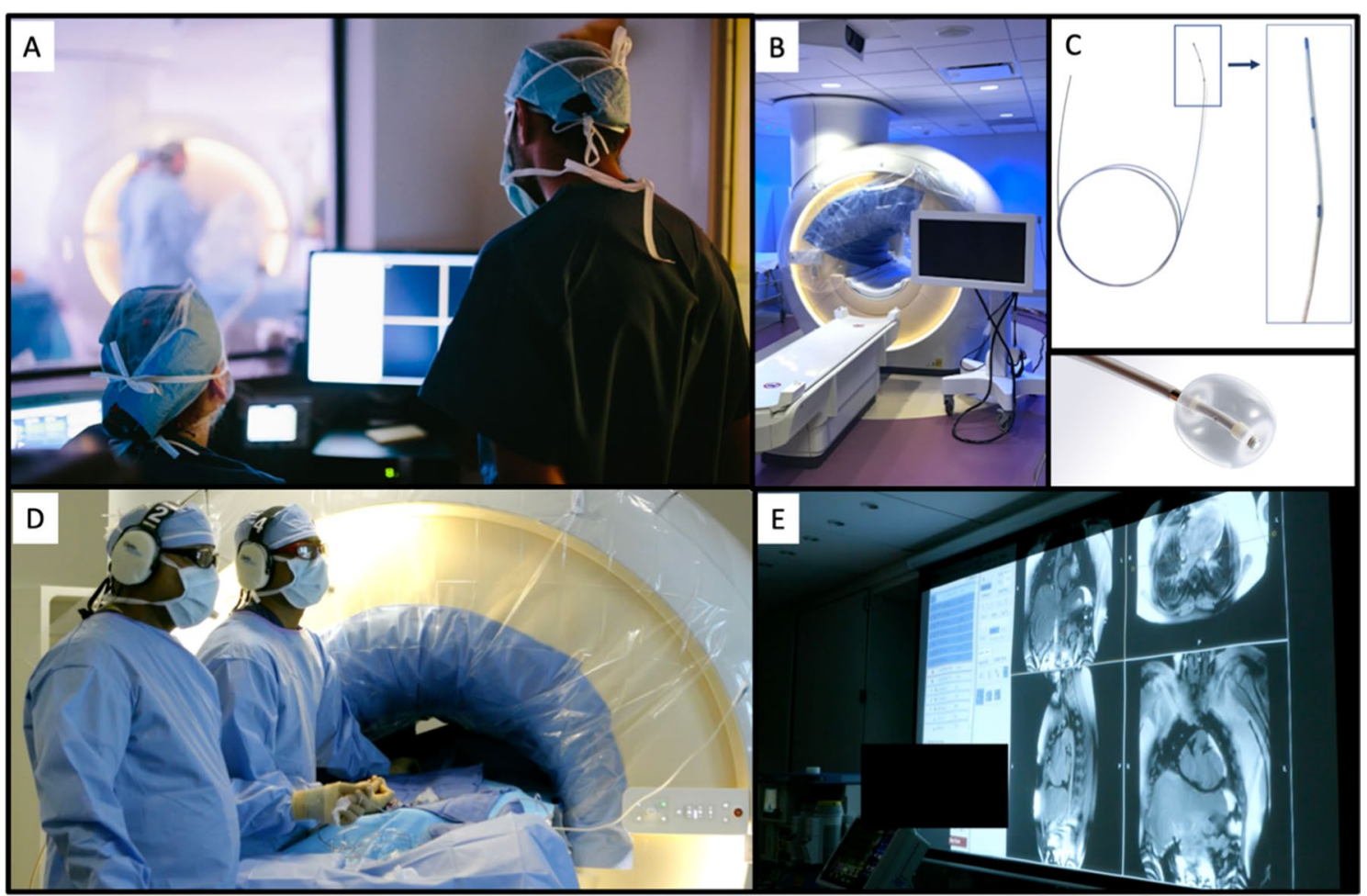

Fig. 1 Invasive cardiovascular magnetic resonance (iCMR) environment and equipment. A CMR team (zone 3) adjusting images with direct visualization of the interventionalist performing the iCMR procedure. B Depiction of sterile draping within the iCMR environment. C Interventionalist equipment includes an MR-conditional catheter and guidewire. The FDA cleared and CE marked guidewire has three passive markers, coated with nanoparticles, that produces a distinct susceptibility artifact $(0 \mathrm{~mm}, 20 \mathrm{~mm}$, and $40 \mathrm{~mm}$ from the tip). D Interventionalist performing an iCMR procedure (zone 4) with real-time CMR guidance on adjacent projector screen (E)

oxygen) under general anesthesia in Zone 4 (actual magnet room) from which all ferromagnetic objects must be excluded [3]. All monitoring is MR-conditional throughout this procedure.

\section{Study methods}

Subjects with CHD underwent real-time $1.5 \mathrm{~T}$ iCMR between March, 29th 2018 to May, 2nd 2019. A recently developed novel passive catheter tracking technique using a real-time single-shot balanced steady-state free precession (bSSFP) with flip angle (FA) $35^{\circ}$, echo time (TE) $1.3 \mathrm{~ms}$, repetition time (TR) $2.7 \mathrm{~ms}$, and a nonselective partial saturation (pSAT) pre-pulse $[3,6]$ was used to visualize the gadolinium-filled balloon, MR-conditional guidewire, and cardiac structures simultaneously to aid in completion of right heart catheterization (RHC) and LHC/aortic pull back under real-time iCMR visualization [3].

Phase contrast CMR (PC-CMR) was used to measure Qp and Qs blood flow. PC images were acquired over 40 cardiac phases, $\mathrm{TE} / \mathrm{TR}=2.7 / 4.4 \mathrm{~ms}$, with two signal averages during free breathing, $2 \times 2 \times 8 \mathrm{~mm}$ resolution, SENSE acceleration factor $=2$, with the velocity encoding gradient set to $25 \%$ above the expected maximum velocity in each vessel. Vendor-provided background phase correction was used, and post-processing was performed using cvi42 (Circle Cardiovascular Imaging Inc., Calgary, Alberta, Canada).

A series of two conditions were performed while the patient was mechanically ventilated on room air to evaluate intra- and inter-rater reliability (Fig. 2). The first condition (C1) was baseline catheter-based Fick and CMR-based arterial flow patterns using PC-CMR. The second condition (C2) is performed under the same clinical settings with no change in ventilation, oxygenation, or hemodynamics. Vitals were monitored and a blood gas was drawn between conditions to ensure steady state of the subject. Extreme care was taken to keep the total fluid status the same during $\mathrm{C} 1$ and $\mathrm{C} 2$. Total flush for catheters was very minimal (total $\sim 10-15 \mathrm{ml}$ ) between sampling measurements. The same $\mathrm{VO}_{2}$ and blood hemoglobin is used for both conditions. Depending on the subject's anatomy and physiology the saturations and pressures obtained differed slightly. In general, we obtained saturations in the conduit, superior vena cava, branch PAs, left atrium (LA)/PVs, and femoral artery. 

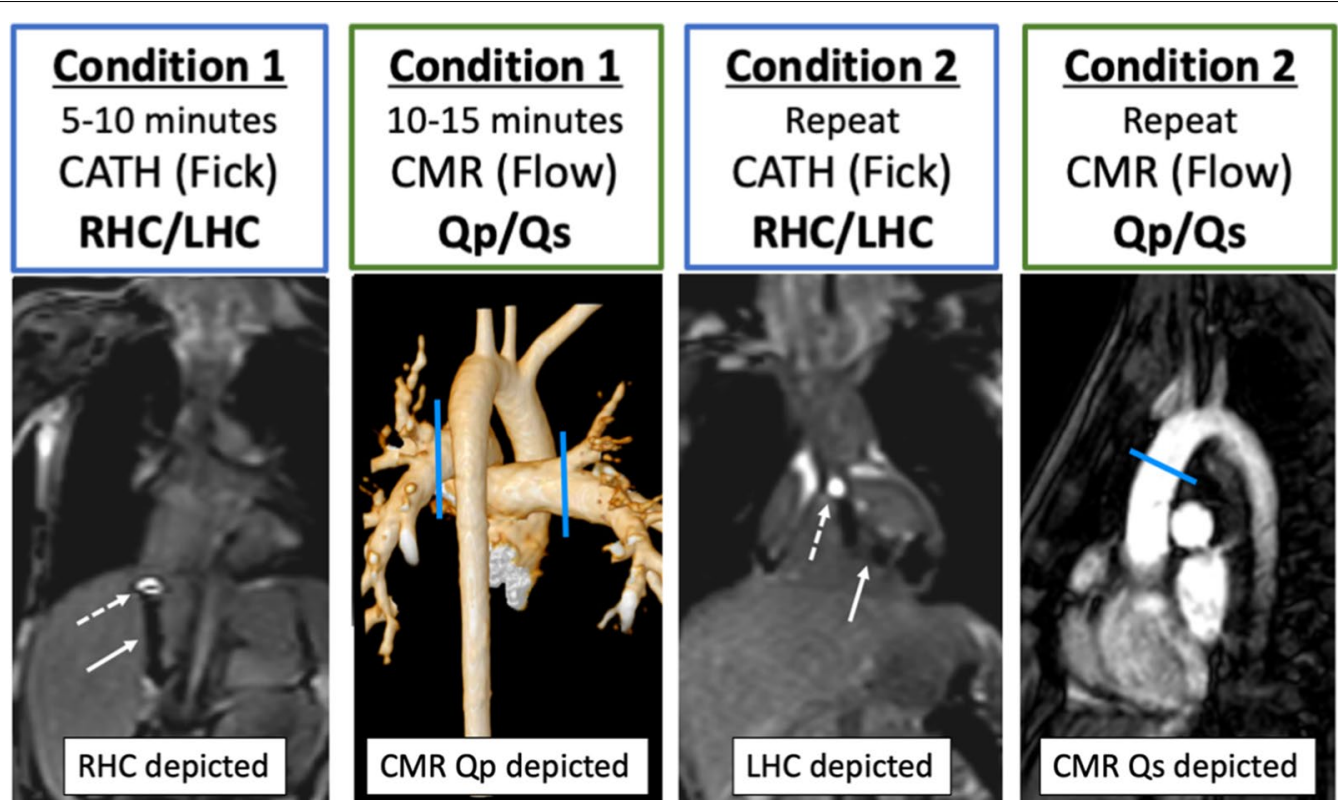

Fig. 2 Test-retest catheter-based Fick and CMR-based flow reliability. A series of two conditions were performed to evaluate intra- and inter-rater reliability between catheter-based Fick and CMR-derived flow hemodynamics. The first condition was baseline catheter-based Fick (right heart catheterization (RHC)/left heart catherization (LHC)) and CMR-based flow (pulmonic flow (Qp)/systemic flow (Qs)) measurements. The second condition was repeat measurement under the same conditions (Cath: RHC/LHC+CMR: Qp/Qs flows). Dashed white arrow - Gadolinium-filled balloon; Solid white arrow - MR-conditional guidewire; Blue line -CMR flow vector

Pressures were obtained in the inferior vena cava (IVC)/ superior vena cava (SVC), hepatic vein with wedge pressures, conduit, branch PA with wedge pressures, right atrium (RA), LA, right ventricle (RV), left ventricle (LV), ascending aorta, descending aorta, and femoral artery. For example, a patient with Fontan completion would have pressure/saturation measurements obtained in the IVC, conduit, SVC, branch PAs, and femoral artery. If a Fontan patient had a patent fenestration then we would attempt to obtain saturations in the common atrium/PVs as well. Similar to standard catheterization lab practices, single sample measurements were obtained in $\mathrm{C} 1$ and repeated again in $\mathrm{C} 2$. Average time between the start of $\mathrm{C} 1$ and the end of $\mathrm{C} 2$ was under thirty minutes.

\section{Statistical considerations}

The Pearson correlation coefficient (PCC) is used to measure test-retest reliability and the concordance correlation coefficient (CCC) is used to quantify agreement between catheter-based Fick and CMR-based flow measurements [7]. Descriptive analyses of the continuous/categorical data were performed using means, confidence intervals, proportions and frequencies. This data was displayed on Bland-Altman and scatter plots (GraphPad Prism, version 8.0.0, GraphPad Software, San Diego, California, USA).

\section{Results}

\section{Patient demographics}

Thirty CHD (21 male) subjects participated in the iCMR reproducibility study at our institution (Fig. 3). Median age and weight were 8.3 years and $27.7 \mathrm{~kg}$ (range: 2-33 yrs and 9.2-80 kgs), respectively. Twenty subjects had single ventricle anatomy with 10 pre-Fontan and 10 postFontan evaluations for protein losing enteropathy (PLE) and/or cyanosis. Ten subjects had bi-ventricular (BiV) anatomy, four were referred for coarctation of the aorta (CoA) evaluations, 3 underwent vaso-reactivity testing with inhaled nitric oxide (iNO), one had multiple cardiac shunts, one underwent branch PA stenosis evaluation, and the remaining subject was status post heart transplant.

\section{Procedural results}

Real-time iCMR-guided RHC (30/30 subjects, 100\%), retrograde and prograde LHC/aortic pull back (30/30 subjects, $100 \%)$ were successfully performed. Total success rate to obtain required data points to complete Fick principle calculations for all patients was 321/328 (98\%). No catheter related complications were encountered. Average time taken for first pass RHC and LHC/aortic pull back was 5.1, and $2.9 \mathrm{~min}$, respectively. One patient with multiple shunts (atrial septal defect, ventricular septal defect, and patent ductus arteriosus) who was referred 


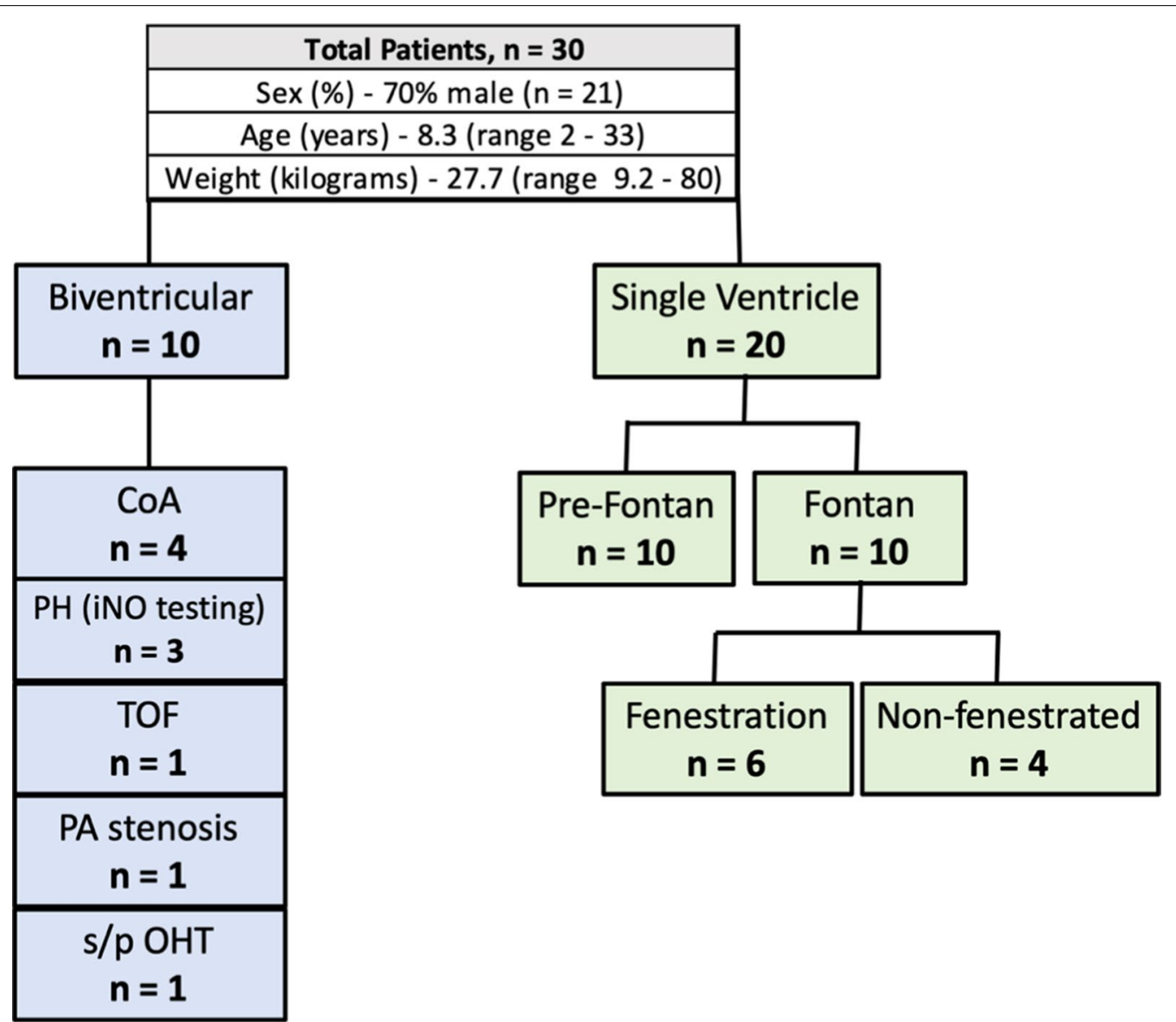

Fig. 3 Basic subject demographics. (CoA=Coarctation of the aorta; $\mathrm{PH}=$ Pulmonary hypertension; $\mathrm{NO}=$ Inhaled Nitric Oxide; TOF = tetralogy of Fallot; $\mathrm{PA}=$ Pulmonary artery; $\mathrm{OHT}=$ Orthotopic heart transplantation)

for vaso-reactivity testing was deemed to be an outlier and excluded from the study's analysis of PCC, CCC, and coefficient of determination. Comparison of invasive catheterization versus iCMR hemodynamic measurements mean and standard deviations are outlined in Fig. 4.

\section{Test-retest reliability}

The PCC for variables measured are shown in Fig. 5 with a Bland-Atlman plot. The PCC for catheter-derived Qp $(0.89, \mathrm{p}<0.001)$ is lower than CMR-derived Qp $(0.96, \mathrm{p}<0.001)$. The PCC for catheter derived Qs $(0.62$, $\mathrm{p}<0.001)$ was considerably lower than CMR derived Qs $(0.94, \mathrm{p}<0.001)$.

\section{Cath and CMR agreement}

Figure 6 is a Bland-Atlman plot of the CCC results. There was fair agreement $(\mathrm{CCC}>0.8)$ for $\mathrm{Qp}$ between catheterization and CMR measurements at baseline $(\mathrm{C} 1-\mathrm{CCC}=0.65,95 \% \mathrm{CI} 0.41-0.81)$, and retested conditions $(\mathrm{C} 2-\mathrm{CCC}=0.78,95 \%$ CI $0.58-0.89$ ).

\section{iCMR scatter plot}

Figure 7 depicts Qp and Qs by graphing an iCMR scatter plot with cath Fick calculations on the $\mathrm{x}$-axis and CMR flows on the $\mathrm{y}$-axis. In addition, $\mathrm{C} 1$ and $\mathrm{C} 2$ as well as single ventricle and biventricular patients are separated to further illustrate the potential differences in each patient population. $\mathrm{C} 1$ and $\mathrm{C} 2$ for each subject is connected with a black line. The patient's with assumed VO2 were outlined in red to help reduce any confounding variables.

\section{Coefficient of determination}

Catheterization, CMR, and comparisons were summarized in Table 1 with coefficients of determination. Qs coefficient of determination for CMR-based flows $\left(R^{2}=0.88\right)$ was found to be much higher than the catheter-derived method $\left(R^{2}=0.39\right)$. 


\begin{tabular}{|c|c|c|c|c|c|}
\hline C1 (Cath) & Mean & Std Dev & C1 (CMR) & Mean & Std Dev \\
\hline Qp & 3.04 & 1.32 & Qp & 3.46 & 1.91 \\
\hline Qs & 3.13 & 0.89 & Qs & 3.43 & 0.94 \\
\hline PVR & 2.04 & 1.94 & PVR & 2.15 & 1.93 \\
\hline SVR & 18.65 & 6.23 & SVR & 17.36 & 7.28 \\
\hline C2 (Cath) & Mean & Std Dev & C2 (CMR) & Mean & Std Dev \\
\hline Qp & 3.13 & 1.28 & Qp & 3.44 & 1.61 \\
\hline Qs & 3.49 & 1.34 & Qs & 3.49 & 1.11 \\
\hline PVR & 2.05 & 1.9 & PVR & 2.23 & 2.23 \\
\hline SVR & 17.45 & 7.12 & SVR & 17.13 & 7.52 \\
\hline
\end{tabular}

Fig. 4 Summary of basic iCMR hemodynamics. Comparison of mean and standard deviation (std dev) measurements for catheterization and CMR hemodynamics for condition 1 (C1) and condition 2 (C2). Qp = Pulmonary blood flow; Qs = Systemic blood flow

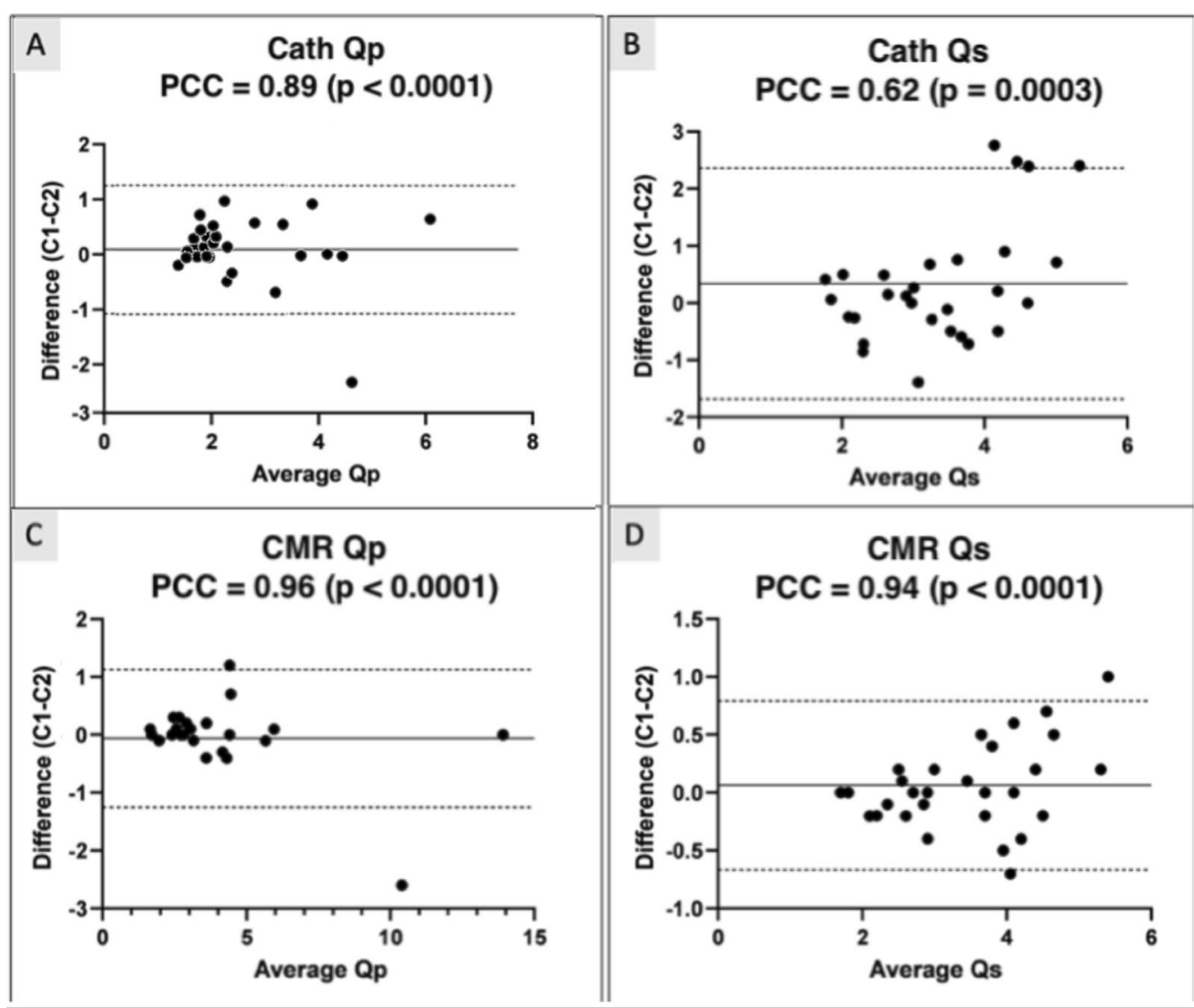

Fig. 5 iCMR intra-rater reliability testing. Bland-Altman plots depicting Pearson correlation coefficients (PCC) to measure test-retest reliability testing between conditions 1 (C1) and 2 (C2) for catheter-based Fick hemodynamics for (A) Qp (B) Qs and CMR-derived flow hemodynamics for (C) Qp and (D) Qs. Qp = Pulmonary blood flow; Qs = Systemic blood flow 

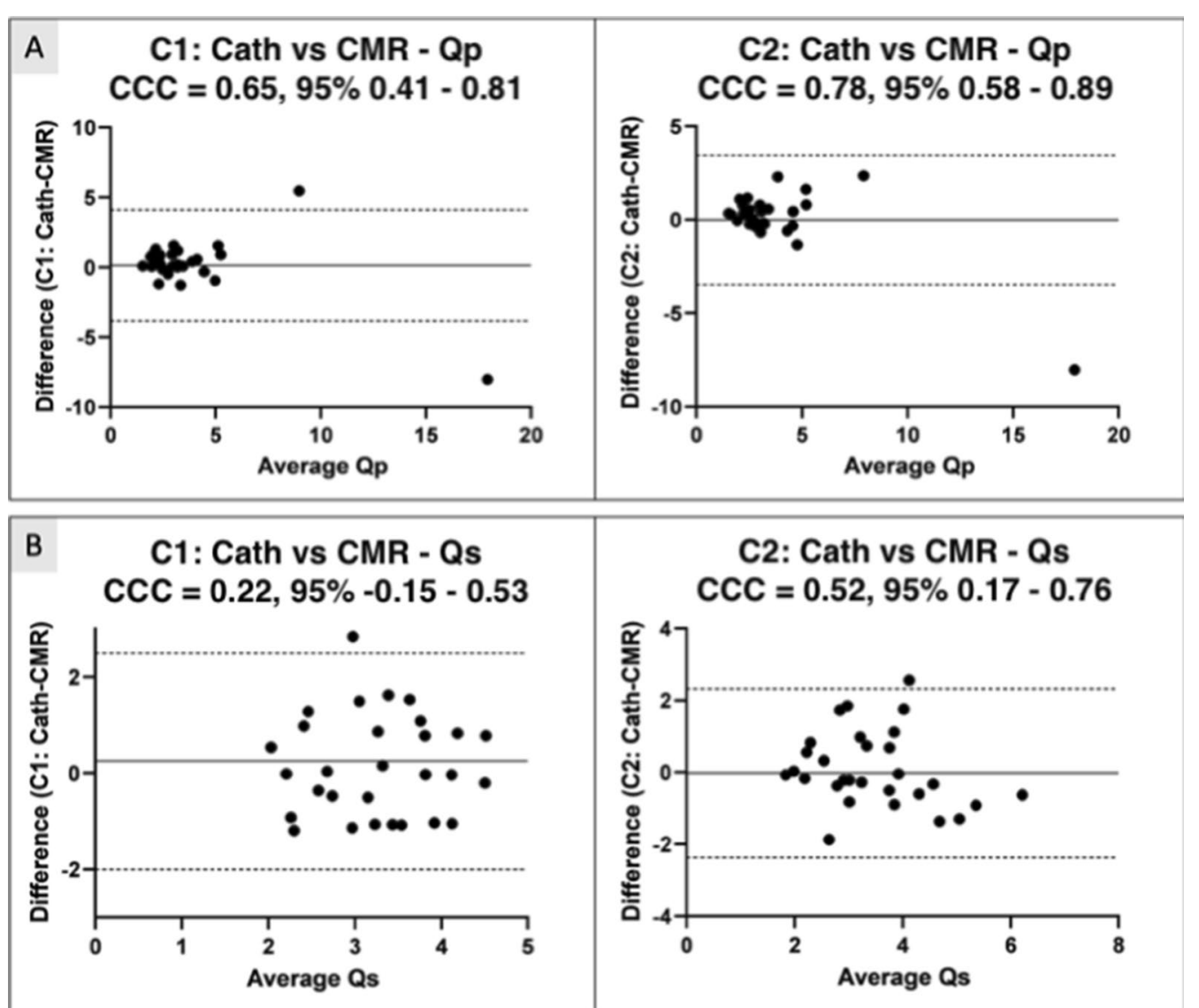

Fig. 6 iCMR inter-rater reliability testing. Bland-Altman plots depicting concordance correlation coefficient (CCC) to measure agreement between catheter-based Fick and CMR-derived flow hemodynamics in condition 1 (C1) and condition 2 (C2) for (A) Qp and (B) Qs. Qp = Pulmonary blood flow; Qs = Systemic blood flow

\section{Discussion}

In this novel iCMR study, we compared and assessed real-time catheter-based Fick principle to CMR-derived flow measurements. Test-retest PCC reliability results for CMR were similar for all variables $(\mathrm{Qp}, \mathrm{Qs} ; \mathrm{p}<0.001)$. However, test-retest PCC reliability results for catheterization were similar only for $\mathrm{Qp}(\mathrm{p}<0.001)$, whereas findings were lower for $\mathrm{Qs}(\mathrm{p}=0.003)$. CMR-derived Qs is significantly more dependable than the catheterbased method. This finding can have significant consequences in a clinician's ability to properly treat and counsel patients. Interestingly, there is good agreement between catheterization and CMR methods to determine PVR which is critically important for the single ventricle population. CMR alone is not able to determine PVR due to the inability to measure pressure non-invasively, with current standard techniques. iCMR PVR is calculated by using the transpulmonary gradient from direct catheter measurements during the same preload and afterload conditions. In an effort to further reduce radiation exposure, $\mathrm{iCMR}$ is emerging as a powerful modality in the assessment of complex CHD.

Previous studies have attempted to compare catheter and CMR-based hemodynamics, however, methods have been limited given the inability to measure right and left sided hemodynamics at the same time under the same conditions [8-12]. In some cases, hemodynamic comparisons between catheterization and CMR were performed months apart. One study specifically looked at subjects with a Glenn circulation $(n=30)$ who underwent catheterization and CMR (XMR) under the same anesthesia [13]. The authors concluded that catheter-derived Fick measurements are generally unreliable in patients with a Glenn circulation. They noted poor correlation when comparing catheter-based Fick principle to the CMR-based flow method ( $\mathrm{Qp}-\rho \mathrm{c}=0.22$; $\mathrm{Qs}-\rho \mathrm{c}=0.24)$. Fick consistently underestimated $Q p$ and overestimated PVR when compared to CMR. In contrast, Fick calculations of Qs overestimate CMR-measured Qs. Another single center study [14] compared these modalities for 

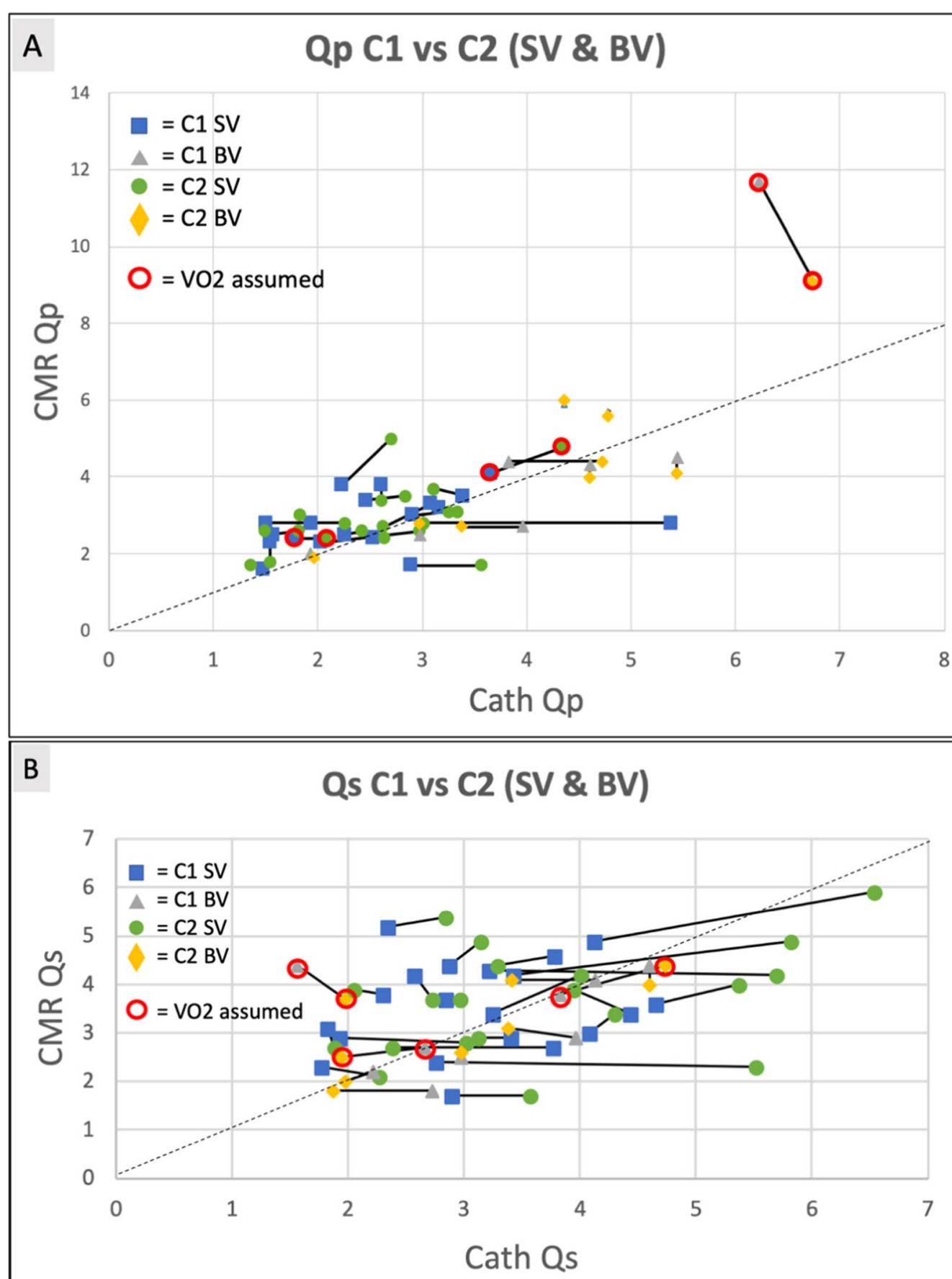

Fig. 7 iCMR flow scatter plots. Comparison of Cath vs CMR blood flow measurements for (A) Qp and (B) Qs. Subjects are grouped based on their underlying anatomy (single ventricle and biventricular). C1 and C2 for each subject is connected by a black line. The dashed line represents an ideal linear relationship. The subjects where VO2 was assumed are outlined in red. Qp = Pulmonary blood flow; Qs = Systemic blood flow; SV = Single ventricle; $\mathrm{BV}=$ Biventricular; $\mathrm{Cl}=$ Condition $1 ; \mathrm{C} 2=$ Condition 2; $\mathrm{VO} 2=$ Oxygen consumption

pre-Glenn and pre-Fontan patients who had a catheterization and $\mathrm{CMR}<1$ month apart $(\mathrm{n}=26)$. They noted that measurements between the two modalities should not be used interchangeably (Qp: $r=0.04, p=0.86$; Qs: $r=0.44, p=0.02$ ), with potential clinical significance in estimating PVR.

Another important study by Rogers et al. [15] successfully recruited 102 patients for real-time CMR 
Table 1 iCMR coefficient of determination

\begin{tabular}{|c|c|c|c|c|c|c|c|c|}
\hline \multicolumn{9}{|c|}{ Linear regression model $n=29$} \\
\hline & $\mathrm{X}=$ Cath C1 & & $\mathrm{X}=\mathrm{CMR} \mathrm{C} 1$ & & $\mathrm{X}=\mathrm{CMR} \mathrm{C} 1$ & & $\mathrm{X}=\mathrm{CMR} \mathrm{C2}$ & \\
\hline & $\mathrm{Y}=$ Cath C2 & & $\mathrm{Y}=\mathrm{CMR} \mathrm{C2}$ & & $\mathrm{Y}=$ Cath C1 & & $\mathrm{Y}=$ Cath $\mathrm{C2}$ & \\
\hline & $Y=$ slope $^{*} x+$ intercept & $R^{2}$ & $Y=$ slope $^{*} x+$ intercept & $R^{2}$ & $Y=$ slope $^{*} x+$ intercept & $R^{2}$ & $Y=$ slope $^{*} x+$ intercept & $R^{2}$ \\
\hline$Q_{p}$ & $0.86 x+0.51$ & 0.80 & $0.79 x+0.69$ & 0.92 & $0.50 x+1.32$ & 0.51 & $0.65 x+0.36$ & 0.65 \\
\hline$Q_{s}$ & $0.94 x+0.54$ & 0.39 & $1.09 x-0.23$ & 0.88 & $0.22 x+2.39$ & 0.05 & $0.62 x+1.34$ & 0.28 \\
\hline
\end{tabular}

Overall coefficient of determination using a linear regression model is calculated between Cath, CMR, and the two method for C1 and C2

Qp, Pulmonary blood flow; Qs, Systemic blood flow; Condition 1; C2, Condition 2

fluoroscopy guided RHC. They showed high rates of procedural success, excellent safety outcomes, and compared cardiac output and PVR quantifications between CMR and cath. However, a major limitation was the inability to perform intra- and/or inter-rater reliability due to the lack of repeatability in their procedural protocol. Our study focused on evaluating the reproducibility of catheter derived Fick calculations versus CMR based flow calculations and assessing the agreement between the two modalities in patients under the same preload and afterload conditions and with all measurements obtained within $30 \mathrm{~min}$ from first sample collection. We showed good CCC $(>0.8)$ implying a good agreement between catheter-based Fick and CMR-based flow measurements for Qp at baseline $(\mathrm{C} 1)$ and retested $(\mathrm{C} 2)$ conditions. With the new FDA-cleared MR-conditional guidewire, we were also able to reliably perform a diagnostic LHC within the CMR-magnet. However, we noted there was poor agreement between the two modalities for Qs assessment at baseline $(\mathrm{C} 1)$ and retested $(\mathrm{C} 2)$ conditions. iCMR enables clinicians to determine a more thoughtful and accurate hemodynamic evaluation in the CHD population. Additional studies are needed to correlate findings between single ventricle and $\mathrm{BiV}$ patients in the iCMR environment including effect of aortopulmonary collaterals burden on Qp, Qs, PVR, and SVR.

Determining PVR remains critically important to risk stratify single ventricle patients for possible catheter and/or surgical interventions. Eligibility for single ventricle palliation is largely determined by direct catheter-based pressure measurements. CMR remains limited by the inability to directly measure vessel and chamber pressures. iCMR is a step toward a more thorough understanding of a patient's true hemodynamics. Our institution has established a set criteria for pre-Glenn patients where CMR alone is appropriate to proceed with single ventricle palliation [16]. Given the ongoing debate for optimal pre-surgical screening for single ventricle palliation, iCMR evaluations will allow clinicians to continue to grow in their understanding of the overlapping utility of this combined iCMR modality.

\section{Limitations}

Our study limitations include a relatively small sample size and combining single ventricle/biventricular subjects, however, the aim of this study was to better understand the test-retest reliability of catheter-based Fick and CMR-based flow measurements across CHD physiologies under the same physiological conditions at the same time. Another potential limitation of the iCMR procedure is the inconsistent visualization of the gadolinium-filled balloon catheter. This issue has been improved by the use of a real-time pSAT sequence described by Velasco Forte et al. [6]. This sequence has been adapted for simultaneous visualization of the gadolinium-filled balloon, MR-conditional guidewire, and cardiac structures as described by Veeram Reddy et al. [3]. In addition, iCMR continues to be extremely limited by the available MR-safe and/or compatible equipment. It is often difficult to enter small and/or stenotic vessels in the CHD population due to the lack of versatility in equipment. It is our hope that industry will be encouraged to fill the obvious device gap in the iCMR arena. Without the manufacturing of a more diverse set of wires and catheters, the field will continue to be limited to routine diagnostics and simple interventions.

Furthermore, the single ventricle population is uniquely positioned to benefit from this modality. We are performing Fontan fenestration test occlusions within the iCMR environment. An iCMR evaluation provides a more critical evaluation of Fontan pressures and Qs at the time of Fontan fenestration test occlusion before referral for fenestration device closure [3, 17]. By using accurate Qs flow measurements with simultaneous catheter-based pressure measurements, the clinician will become more confident to make an informed decision for Fontan fenestration device closure. 
In addition, CMR provides precise anatomical and functional data to plan complex CHD interventions $[18,19]$. In recent years, congenital cardiology has been investigating an exponential increase in the indications for CMR to answer significant clinical questions that are not able to be assessed by other imaging modalities alone. We continue to investigate non-invasive measurements of blood oxygen saturation, PVR, and APC burden. The iCMR environment allows for a more critical evaluation of complex CHD subjects by allowing for assessment of transcatheter pressures during CMR derived flow measurements.

\section{Conclusion}

Real-time, radiation-free iCMR-guided cardiovascular catheterizations allows for simultaneous measurement of catheter-based hemodynamics and CMR-derived flows to quantify and compare important hemodynamic variables including Qp and Qs. Most notably, CMR-derived flows have higher test-retest reliability for Qs when compared to catheter-based Fick principle. iCMR evaluations allow for more reproducible hemodynamic assessments in the CHD population. This pilot study further endorses non-invasive diagnostic parameters to better serve the $\mathrm{CHD}$ population in the planning stages prior to catheterbased and/or surgical interventions.

\begin{abstract}
Abbreviations
BiV: Biventricular; bSSFP: Balanced steady state free precession; CHD: Congenital heart disease; CMR: Cardiovascular magnetic resonance; CoA: Coarctation of the aorta; FDA: United States Food and Drug Administration; iCMR: Invasive cardiovascular magnetic resonance; iNO: Inhaled nitric oxide; IVC: Inferior vena cava; LA: Left atrium/left atrial; LHC: Left heart catheterization; LV: Left ventricle/left ventricular; MV: Mixed venous; PA: Pulmonary artery; PC: Phase contrast; PCC: Pearson correlation coefficient; PH: Pulmonary hypertension; pSAT: Partial saturation; PV: Pulmonary vein; PVR: Pulmonary vascular resistance; Qp: Pulmonary blood flow; Qs: Systemic blood flow; RA: Right atrium/ right atrial; RHC: Right heart catheterization; RV: Right ventricle/right ventricular; SVC: Superior vena cava; SVR: Systemic vascular resistance; TOF: Tetralogy of Fallot; TPG: Transpulmonary pressure gradient; TSG: Transsystemic gradient; VO2: Oxygen consumption.
\end{abstract}

\section{Acknowledgements}

We would like to acknowledge the help of King's College London Biomedical Engineer's work on PSAT, Nano4Imaging's Phantom device and MR-conditional guidewire, and Dr. John Kakareka for PRiMEGen installation.

\section{Disclosures}

Whilst Nano4lmaging did not provide any financial support for this study, they have provided travel expenses for the senior author (SRVR) for attendance at an international conference to present related work.

\section{Authors' contributions}

YA, TH, RAZ, VG, JSG, RH, JH, JK, GG and SRVR were involved with project design, data analysis, drafting the article, and critical revision of the article. All authors reviewed the manuscript and agree with its publication. All authors read and approved the final manuscript.

\section{Funding}

Research reported in this publication was supported by Children's Health, Dallas and the Moss Foundation. The content is solely the responsibility of the authors and does not necessarily represent the official views of Children's Health. The Pogue Family Distinguished Chair in Pediatric Cardiology was awarded to Dr. F. Gerald Greil in February 2015. The purpose of the funds is to encourage research, personnel and institution development. Pogue Family Distinguished Chair - Dr. Gerald Greil. Children's Clinical Research Advisory Committee - Dr. Yousef Arar. Harry S. Moss Foundation - Dr. Tarique Hussian.

\section{Availability of data and materials}

The datasets used and/or analyzed during the current study are available from the corresponding author on reasonable request.

\section{Declarations}

\section{Ethics approval and consent to participate}

Approval was obtained from the local ethics committee. The UT Southwestern IRB approved STU 032017-061. All subjects/ legal guardians gave written consent and assent as appropriate.

\section{Consent for publication}

The UT Southwestern IRB approved the use of images and content from this study and waived the need for consent, STU 032017-061.

\section{Competing interests}

Whilst Nano4lmaging did not provide any financial support for this study, they have provided travel expenses for the senior author (SR) for attendance at an international conference to present related work.

\section{Author details}

${ }^{1}$ Department of Pediatrics, University of Texas Southwestern Medical Center, Dallas, TX, USA. ${ }^{2}$ Department of Radiology, University of Texas Southwestern Medical Center, Dallas, TX, USA. ${ }^{3}$ Pediatric Cardiology, Children's Medical Center, 1935 Medical District Drive, Dallas, TX 75235, USA. ${ }^{4}$ Research Administration, Children's Medical Center, Dallas, TX, USA. ${ }^{5}$ Anesthesiology and Pain Management, Children's Medical Center, Dallas, TX, USA.

Received: 26 June 2020 Accepted: 26 May 2021

Published online: 19 July 2021

\section{References}

1. Fogel MA, Pawlowski TW, Whitehead KK, Harris MA, Keller MS, Glatz AC, Zhu W, Shore D, Diaz LK, Rome JJ. Cardiac magnetic resonance and the need for routine cardiac catheterization in single ventricle patients prior to Fontan: a comparison of 3 groups: pre-Fontan CMR versus cath evaluation. J Am Coll Cardiol. 2012;60(12):1094-102. https://doi.org/10.1016/j. jacc.2012.06.021.

2. Brown DW, Gauvreau K, Powell AJ, Lang P, Del Nido PJ, Odegard KC, Geva T. Cardiac magnetic resonance versus routine cardiac catheterization before bidirectional Glenn anastomosis: long-term follow-up of a prospective randomized ) trial. J Thorac Cardiovasc Surg. 2013. https:// doi.org/10.1016/j.jtcvs.2012.12.079.

3. Veeram Reddy SR, Arar Y, Zahr RA, et al. Invasive cardiovascular magnetic resonance (iCMR) for diagnostic right and left heart catheterization using an MR-conditional guidewire and passive visualization in congenital heart disease. J Cardiovasc Magn Reson. 2020;22:20. https://doi.org/10. 1186/s12968-020-0605-9.

4. Kakareka JW, Faranesh AZ, Pursley RH, Cambell-Washburn A, Herzka DA, Rogers T, Kanter J, Ratnayaka K, Lederman RJ, Pohida TJ. Physiological recording in the MRI environment (PRIME): MRI-compatible hemodynamic recording system. IEEE J Transl Eng Health Med. 2018;6:1.

5. LaFarge CG, Miettinen OS. The estimation of oxygen consumption. Cardiovasc Res. 1970;4(1):23-30.

6. Velasco Forte MN, Pushparajah K, Schaeffter T, Valverde Perez I, Rhode K, Ruijsink B, Alhrishy M, Byrne N, Chiribiri A, Ismail T, Hussain T, Razavi R, Roujol S. Improved passive catheter tracking with positive contrast for CMR-guided cardiac catheterization using partial saturation (pSAT). J Cardiovasc Magn Reson. 2017. https://doi.org/10.1186/s12968-017-0368-0.

7. Lin LI. A concordance correlation coefficient to evaluate reproducibility. Biometrics. 1989;45:255-68. 
8. Rogers T, Ratnayaka K, Khan JM, Stine A, Schenke WH, Grant LP, Mazal JR, Grant EK, Campbell-Washburn A, Hansen MS, Ramasawmy R, Herzka DA, Xue H, Kellman P, Faranesh AZ, Lederman RJ. CMR fluoroscopy right heart catheterization for cardiac output and pulmonary vascular resistance: results in 102 patients. J Cardiovascr Magn Res. 2017;19:54.

9. Narang N, Thibodeau JT, Levine BD, Gore MO, Ayers CR, Lange RA, et al. Inaccuracy of estimated resting oxygen uptake in the clinical setting. Circulation. 2014;129:203-10. https://doi.org/10.1161/CIRCULATIONAHA. 113.003334 .

10. Nayak KS, Nielsen JF, Bernstein MA, Markl M, Gatehouse PD, Botnar RM, et al. Cardiovascular magnetic resonance phase contrast imaging. J Cardiovasc Magn Reson. 2015;17:71. https://doi.org/10.1186/ s12968-015-0172-7.

11. Muthurangu V, Taylor A, Andriantsimiavona R, Hegde S, Miquel ME, Tulloh $R$, et al. Novel method of quantifying pulmonary vascular resistance by use of simultaneous invasive pressure monitoring and phase-contrast magnetic resonance flow. Circulation. 2004;110:826-34. https://doi.org/ 10.1161/01.CIR.0000138741.72946.84.

12. Razavi R, Hill DL, Keevil SF, Miquel ME, Muthurangu V, Hegde S, Rhode K, Barnett M, van Vaals J, Hawkes DJ, et al. Cardiac catheterisation guided by MRI in children and adults with congenital heart disease. Lancet. 2003;362(9399):1877-82. https://doi.org/10.1016/S0140-6736(03) 14956-2.

13. Downing TE, Whitehead K, Dori Y, Gillespie M, Harris M, Fogel M, Rome J, Glatz A. Accuracy of conventional oximetry for flow estimation in patients with superior cavopulmonary connection a comparison with phasecontrast cardiac MRI. Circ Cardiovasc Imaging. 2013;6:943-9.

14. Hart MR, Whiteside W, Yu S, Lowery R, Dorfman AL, Mahani MG, Agarwal PP, Lu JC. Differences in pulmonary and systemic flow measurements by cardiac magnetic resonance vs cardiac catheterization and relation to collateral flow in single ventricle patients. Pediatric Cardiol. 2020. https:// doi.org/10.1007/s00246-020-02327-w.

15. Rogers T, Ratnayaka K, Khan JM, Stine A, Schenke WH, Grant LP, Mazal JR, Grant EK, Campbell-Washburn A, Hansen MS, Ramasawmy R, Herzka DA, Xue $H$, Kellman P, Faranesh AZ, Lederman RJ. CMR fluoroscopy right heart catheterization for cardiac output and pulmonary vascular resistance: results in 102 patients. J Cardiovasc Magn Reson. 2017;19:54. https://doi. org/10.1186/s12968-017-0366-2.

16. James L, Tandon A, Nugent A, Malik S, Ramaciotti C, Greil G, Zabala L, Forbess J, Hussain T. Rationalising the use of cardiac catheterisation before Glenn completion. Cardiol Young. 2018;28:719-24. https://doi.org/ 10.1017/S1047951118000240.

17. Arar Y, Hussain T, D, Abou Zahr R, Hernandez J, Blair Z, Greil G, Veeram Reddy SR. Fontan Fenestration Test Occlusion Evaluation in the iCMR Suite. Society for Cardiovascular Magnetic Resonance (SCMR, 2019 abstr).

18. Arar Y, Reddy SRV, Kim H, Dimas W, Zellers TM, Zahr AR, Vamsee R, Greer JS, Tandon A, Pontiki A, Dillenbeck J, Zabala L, Greil G, Nugent AW, Hussain $\mathrm{T}$. 3D advanced imaging overlay with rapid registration in CHD to reduce radiation and assist cardiac catheterisation interventions. Cardiol Young. 2020. https://doi.org/10.1017/S1047951120000712.

19. Ratnayaka K, Rogers T, Schenke WH, et al. Magnetic resonance imagingguided transcatheter cavopulmonary shunt. JACC Cardiovasc Interv. 2016;9:959-70. https://doi.org/10.1016/j.jcin.2016.01.032.

\section{Publisher's Note}

Springer Nature remains neutral with regard to jurisdictional claims in published maps and institutional affiliations.
Ready to submit your research? Choose BMC and benefit from:

- fast, convenient online submission

- thorough peer review by experienced researchers in your field

- rapid publication on acceptance

- support for research data, including large and complex data types

- gold Open Access which fosters wider collaboration and increased citations

- maximum visibility for your research: over 100M website views per year

At BMC, research is always in progress.

Learn more biomedcentral.com/submissions 